\title{
Carboxy-terminus Hsc70 interacting protein exerts a tumor inhibition function in head and neck cancer
}

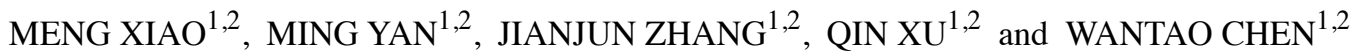 \\ ${ }^{1}$ Department of Oral and Maxillofacial-Head and Neck Oncology, Ninth People's Hospital, Shanghai Jiaotong University, \\ School of Medicine; ${ }^{2}$ Shanghai Key Laboratory of Stomatology and Shanghai Research Institute of Stomatology, \\ Shanghai 200011, P.R. China
}

Received January 31, 2017; Accepted July 7, 2017

DOI: $10.3892 /$ or.2017.5827

\begin{abstract}
Several independent studies have reported the roles of the E3 ubiquitin ligase, carboxy-terminus Hsc70 interacting protein (CHIP) in various types of cancers. However, the biological effects of CHIP vary in regards to different cancers, and the role of CHIP in head and neck cancers (HNCs) remains unknown. In the present study, CHIP overexpression plasmids and CHIP knockdown lentivirus were constructed to affect the expression levels of CHIP protein and biological behaviors in $\mathrm{HNC}$ cell lines bilaterally. The biological behaviors regulated by CHIP in HNCs were investigated both in vivo and in vitro with a series of assays and analyses. A tissue microarray was stained and analyzed for the clinical significance of CHIP expression in HNCs. We identified that CHIP suppressed the malignant behaviors of HNCs in a series of in vitro and in vivo experiments, but not its two loss-of-function mutants. However, we observed an altered expression pattern of CHIP from a well, moderate, to poor differentiation pathological status in HNC specimens. In a retrospective cohort of HNCs, lower expression of CHIP indicated a poor differentiation status in tumors and a lower overall survival rate. The present study demonstrated that CHIP functions as a tumor suppressor in HNCs. In conclusion, we demonstrated that suppressed expression of CHIP may result in the progression of HNCs.
\end{abstract}

\section{Introduction}

The ubiquitin-proteasome system is a major pathway that contributes to intracellular proteostasis. Carboxy-terminus of Hsc70 interacting protein (CHIP) has been identified as an E3 ubiquitin ligase and a potent regulator protein in maintaining protein homeostasis in diverse cellular processes (1-3).

Correspondence to: Dr Wantao Chen, Department of Oral and Maxillofacial-Head and Neck Oncology, Ninth People's Hospital, Shanghai Jiaotong University School of Medicine, 639 Zhizaoju Road, Shanghai 200011, P.R. China

E-mail: chenwantao196323@sjtu.edu.cn

Key words: CHIP, ubiquitin E3 ligase, head and neck cancer, tumor growth inhibition
Structurally, 3 tandem repeats of the tetratricopeptide (TPR) motif and a C-terminal U-box domain (U-box) contribute to the bioactivity of CHIP as a chaperone-associated E3 ubiquitin ligase involved in the ubiquitin-proteasome system $(4,5)$. CHIP post-translationally controls the turnover of its substrate proteins and exerts regulatory roles in a myriad of biological processes $(6,7)$.

Until recently, accumulating evidence has suggested a role for CHIP in the initiation and progression of cancers (7-9). During the development of carcinogenesis, the production of mutated or aberrant cellular proteins must be gradually increased during malignant transformation and in response to genetic instability processes $(10,11)$. As previously reported, CHIP not only induces ubiquitylation and degradation of several oncogenic proteins $(7,12-14)$, but also regulates tumor suppressor proteins $(15,16)$, which has increased interest in understanding the mechanisms of CHIP in the context of carcinogenesis. Accordingly, the diversities of CHIP substrate proteins present heterogeneity among different tissue-derived cancers, and the biological functions of CHIP in head and neck cancers (HNCs) remain unclear.

In the present study, we conducted a comprehensive study on CHIP in HNCs from laboratory experiments to clinical data. Our results showed that CHIP functions as a tumor suppressor in HNC cell lines and a regular pattern of CHIP expression from well, moderate, to poor differentiation state was illustrated. In conclusion, we demonstrated that low expression of CHIP is a potential risk factor for HNC patients.

\section{Materials and methods}

Cell culture and reagents. HNC cell lines (HN13, HN30, Cal27 and UMSCC12) were cultured in Dulbecco's modified Eagle's medium (DMEM; Basal Media, Shanghai, China) supplemented with $10 \%$ fetal bovine serum (FBS; Gibco-Invitrogen, Carlsbad, CA, USA), $100 \mathrm{U} / \mathrm{ml}$ penicillin and $100 \mu \mathrm{g} / \mathrm{ml}$ streptomycin, at $37^{\circ} \mathrm{C}$ in the presence of $5 \%$ carbon dioxide $(17,18)$. Thiazolyl blue tetrazolium bromide (MTT) was purchased from Sigma (St. Louis, MO, USA).

Plasmid constructs and transfections. The mammalian expression vector used was pcDNA3.1(+)-myc-CHIP. Two loss-of-function CHIP mutants were generated using 
the QuickChange XL Site-Directed Mutagenesis kit (Life Technologies, Carlsbad, CA, USA) at the TPR domain (K30A), and U-box domain (H260Q), respectively. The primer sequences used for site-directed mutagenesis were: forward, 5'-GCG CGC AGG AGC TCG CGG AGC AGG GCA ATC-3' and reverse, 5'-GAT TGC CCT GCT CCG CGA GCT CCT GCG CGC-3' for CHIP (K30A); and forward, 5'-ACA TCG AGG AGC AGC TGC AGC GTG TGG-3' and reverse, 5'-CCA CAC GCT GCA GCT GCT CCT CGA TGT-3' for CHIP (H260Q) $(6,16)$. Plasmids were transfected into cells using Lipofectamine 2000 (Invitrogen, Carlsbad, CA, USA) diluted in Opti-MEM ${ }^{\circledR}$ I (Life Technologies) according to the manufacturer's instructions. UMSCC12-shCHIP stable cells were generated by transfecting UMSCC12 cells with the CHIP-specific short hairpin RNA (5'-GGACGA CAT CCC CAG CGC TCT-3') lentivirus and selected with $10 \mu \mathrm{g} /$ $\mathrm{ml}$ of puromycin (Calbiochem, La Jolla, CA, USA) for positive clones (13), and UMSCC12-scrambled cells were used as control.

Cell proliferation analysis. MTT assays were performed to examine the proliferative ability of involved cells in the present study. Absorbance measurements following dimethyl sulfoxide (DMSO) resolution were performed at a wavelength of $490 \mathrm{~nm}$ (Bio-Rad Laboratories, Hercules, CA, USA).

Colony formation assay. Cell suspension was counted, diluted and seeded at $1 \times 10^{3} /$ well into a 6 -well plate in triplicates. Cells were kept culturing for 10 days, after which the cells were fixed with paraformaldehyde and stained with Coomassie Brilliant Blue, subsequently. The number of large colonies was counted and analyzed (18).

Transwell assays. To determine cell migration, $5 \times 10^{4}$ cells suspended in $200 \mu \mathrm{l}$ of fresh DMEM were plated into Millicell chambers ( $8 \mu \mathrm{m}$; Millipore Corp., Billerica, MA, USA) with $600 \mu \mathrm{l}$ of DMEM containing 10\% FBS in the bottom chamber. Cells that migrated through the filter at $24 \mathrm{~h}$ were fixed with paraformaldehyde and stained with $10 \%$ crystal violet. To determine cell invasion, $50 \mu \mathrm{l}$ Matrigel (1:8 diluted; BD Biosciences, Franklin Lakes, NJ, USA) was used to coat the chamber beforehand, and the invaded cells were harvested at $48 \mathrm{~h}$. The cells were photographed, and the number of cells that migrated or invaded were counted based on 5 microscopic $\times 100$ fields/insert.

Flow cytometric analysis. To analyze apoptotic cells, trypsinized adherent and floating cells were harvested and prepared using the FITC/Annexin V Apoptosis Detection Kit I (BD Pharmingen, San Jose, CA, USA) according to the protocols recommended by the manufacturer. After staining, the cells were analyzed using flow cytometry (FACSCaliber; BD Biosciences).

Western blot analysis. Cellular extracts were acquired using whole cell lysis buffer containing proteinase inhibitor cocktail. After subjecting the lysates to SDS-PAGE electrophoresis, proteins were transferred onto a polyvinylidene difluoride (PVDF) membrane by electroblotting. The membranes were then blocked in 5\% skimmed milk for $1 \mathrm{~h}$ and incubated overnight at $4^{\circ} \mathrm{C}$ with specific primary antibodies. Specific antibody-bound protein bands were detected with fluorescent secondary antibody and visualized using an Odyssey Infrared Imaging System (BD Biosciences, San Diego, CA, USA) (17). In the present study, the rabbit monoclonal antibody against CHIP (1:1,000; Abcam, Cambridge, MA, USA) and rabbit polyclonal antibody against Myc Tag (1:1,000, Abbkine, Redlands, CA, USA) were used. The mouse monoclonal against $\beta$-tubulin (1:1,000, Boster, Wuhan, China) served as a loading control.

Animal experiments. Male BALB/c nude mice (nu/nu, aged 4-5 weeks) were purchased from Shanghai Laboratory Animal Center (Shanghai, China), and were housed under specific pathogen-free conditions in the Experimental Animal Care Center of Shanghai Ninth People's Hospital. Animal welfare and experimental procedures were conducted in compliance with the Guide for Care and Use of Laboratory Animals (The Ministry of Science and Technology of China, 2006) and the related ethical regulations of the hospital. The Animal Care and Use Committees of the hospital approved all experimental procedures.

Briefly, the nude mouse xenograft tumor models were established by subcutaneous injection of $5 \times 10^{6}$ cells/site. To evaluate the effects of CHIP knockdown on UMSCC12 tumorigenesis, UMSCC12-scrambled cells were implanted on the right flank, and UMSCC12-shCHIP cells were implanted on the left flank $(n=6)$. Tumor volumes (length $\times$ width $\left.^{2} / 2\right)$ were monitored.

Immunohistochemistry staining. Fresh tissues were fixed in $4 \%$ formaldehyde, embedded in paraffin and prepared into $5-\mu \mathrm{m}$ sections. After dewaxing, rehydration and antigen retrieval, endogenous peroxidase activity was quenched. The slides were incubated with primary antibody overnight at $4^{\circ} \mathrm{C}$. Then, the samples were incubated with a biotinylated secondary antibody for $50 \mathrm{~min}$ at $37^{\circ} \mathrm{C}$, which was followed by staining with a DAB kit (GTVision; China). The rabbit polyclonal antibody against CHIP (1:250; Abcam) and mouse monoclonal antibody against Ki67 (1:500; Santa Cruz Biotechnology, Inc., Santa Cruz, CA, USA) were used as primary antibodies.

Scoring for tissue-array staining. A tissue microarray was constructed using primary HNC samples from 101 patients who received radical surgery, and 10 out-patient biopsy samples for a potential cancer lesion, but pathologically not (3 oral ulcer, 6 oral leukoplakia, and 1 normal oral epithelial sample) from the Department of Oral Maxillofacial-Head and Neck Oncology, Shanghai Ninth People's Hospital (17). The patients involved in the present study signed written informed consent, and the study was approved by the Medical Ethics Committee of the Ninth People's Hospital, Shanghai Jiaotong University School of Medicine.

Three samples were excluded due to lack of tissue or cancer cells in the array. The immunoreactivity score (IRS) for CHIP immunohistochemistry (IHC) staining was recorded by two independent observers, who scored based on staining intensity and percentage of positive cancer cells. The staining intensity was scored as follows: weak, scored 1; moderate, scored 2; and intense, scored 3. Regarding the percentage of 


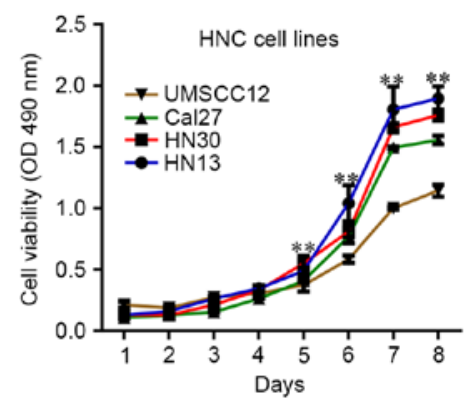

C

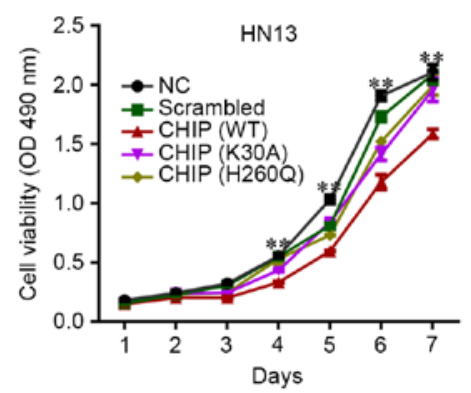

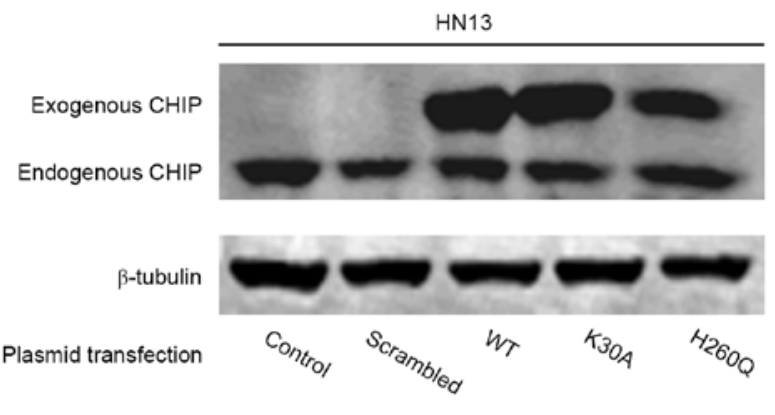

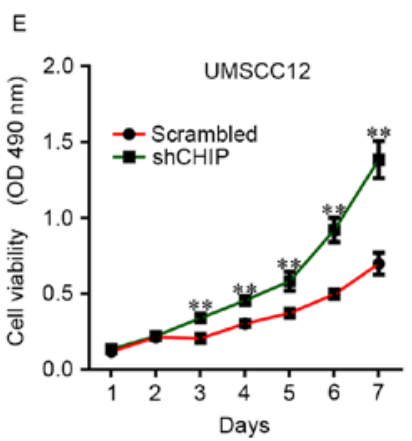

D

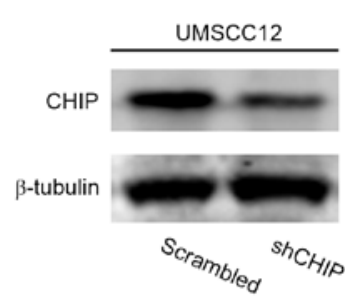

$\mathrm{F}$

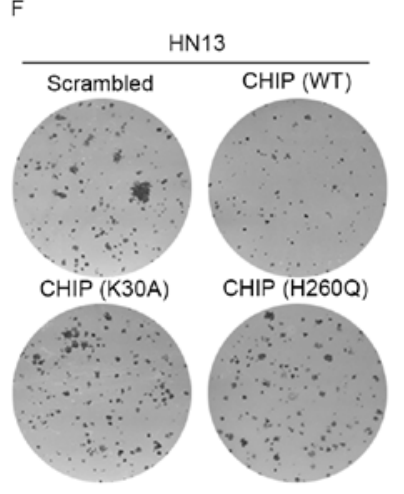

G

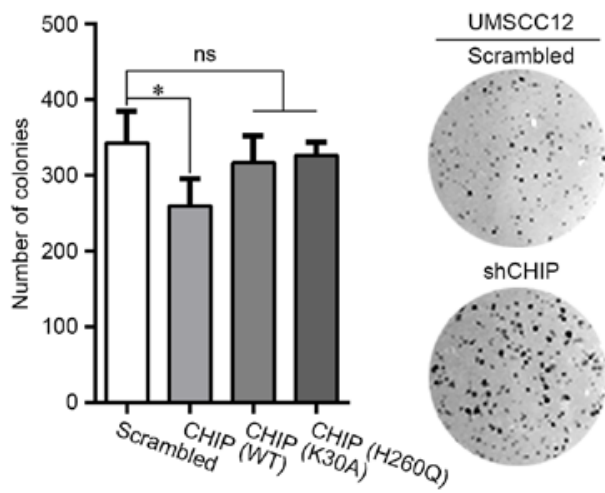

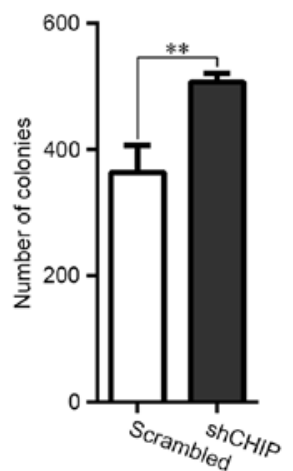

Figure 1. CHIP regulates the proliferative and colony forming abilities of HNC cell lines. (A) The general proliferative potency of HN13, HN30, Cal27 and UMSCC12 cells was measured by MTT assays ( $\left.{ }^{* *} \mathrm{p}<0.01\right)$. (B) Western blot analysis for the transfected efficiency of CHIP and its mutants in HN13 cells. (C) The effect of wild-type CHIP and loss-of-function CHIP mutants on the proliferation of HN13 cells was analyzed with MTT assays (** $<<0.01)$. (D) Western blot analysis for the knockdown efficiency of CHIP expression in UMSCC12 cells. (E) The effect of CHIP knockdown on the proliferation of UMSCC12 cells was analyzed by MTT assays (** $\mathrm{p}<0.01$ ). (F) The effect of wild-type and two loss-of-function CHIP mutants on the colony formation potential of HN13 cells ( $\mathrm{p}=0.039 ; \mathrm{ns}$, not significant). (G) The effect of CHIP knockdown on the colony formation potential of UMSCC12 cells ( $* \mathrm{p}<0.01)$.

positive cancer cells, the score was defined as follows: $0-25 \%$, scored $1 ;>25-50 \%$, scored $2 ;>50-75 \%$, scored $3 ;>75 \%$, scored 4. Finally, an overall score (ranging from 1-12) was acquired by multiplying the above two scores for each sample. A total score of 1-6 was considered low expression; 7-12 was considered high expression.

Statistical analysis. The data were compiled using the software package SPSS, version 12.0 (SPSS, Inc., Chicago, IL, USA). Chi-squared tests were used to assess the statistical significance for correlations between CHIP expression and clinicopathological variables. Univariate survival analysis was performed using the Kaplan-Meier method, and differences in survival curves were assessed by the log-rank test. All experiments were performed in triplicate; and representative results are displayed. The values displayed correspond to the means \pm SD. Significant differences between two groups were determined based on Student's t-test and a p-value $<0.05$ was considered statistically significant.

\section{Results}

Involvement of CHIP expression in the proliferative and colony forming ability of HNCs. By evaluating the cellular proliferation rate of $4 \mathrm{HNC}$ cell lines, we found that HN13 displayed the greatest proliferative capacity, whereas UMSCC12 displayed the least proliferative capacity (Fig. 1A). Next, we managed to overexpress CHIP (CHIP $\left.{ }^{\mathrm{OE}}\right)$ and its two loss-of-function mutants in the $\mathrm{HN} 13$ cells (Fig. 1B). CHIP significantly suppressed cellular proliferation in HN13 cells, whereas overexpression of loss-of-function CHIP mutants (K30A and H260Q) abolished such effects (Fig. 1C). The expression of CHIP in UMSCC12 cells was suppressed by stable transfection with CHIP-specific short hairpin RNA 


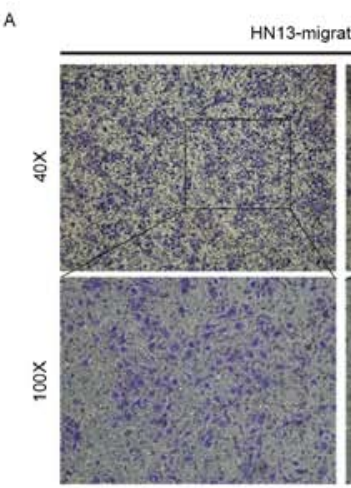

Scrambled

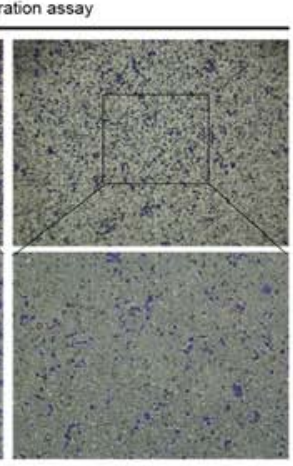

CHIP

B

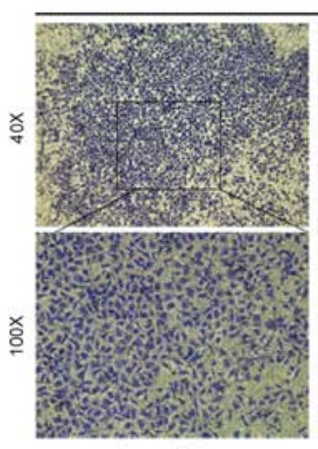

Scrambled

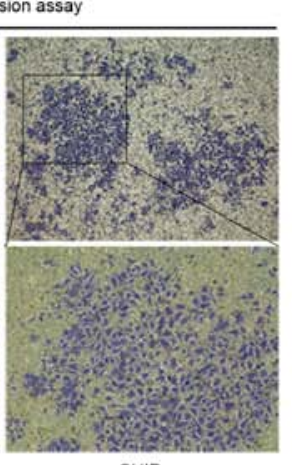

CHIP

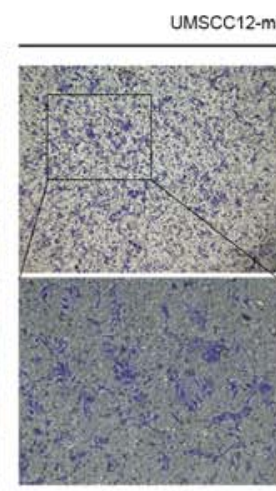

Scrambled

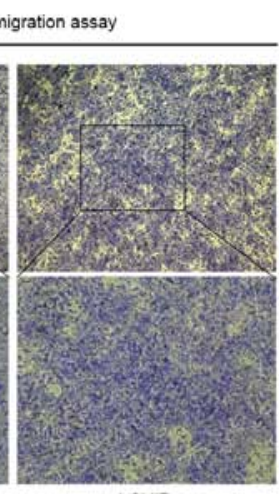

shCHIP

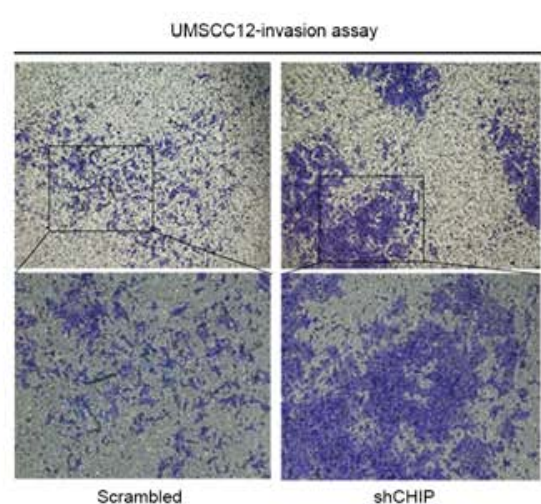

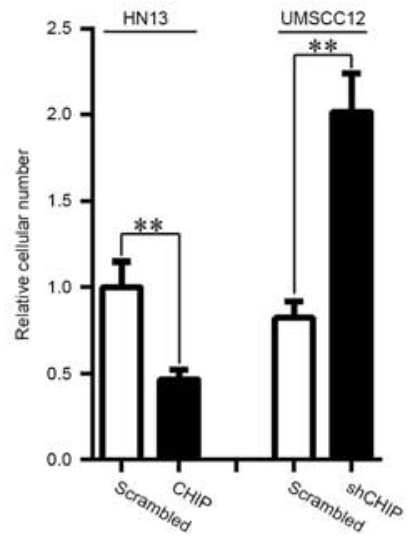

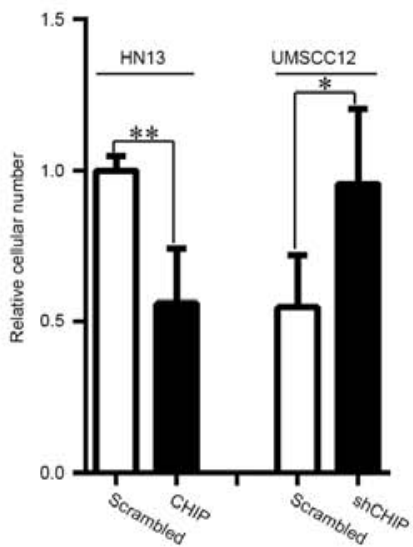

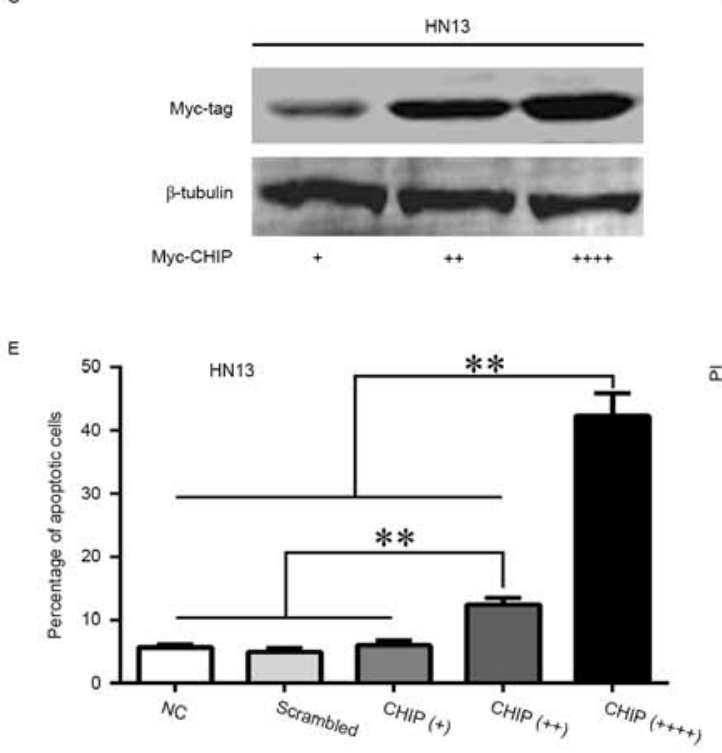

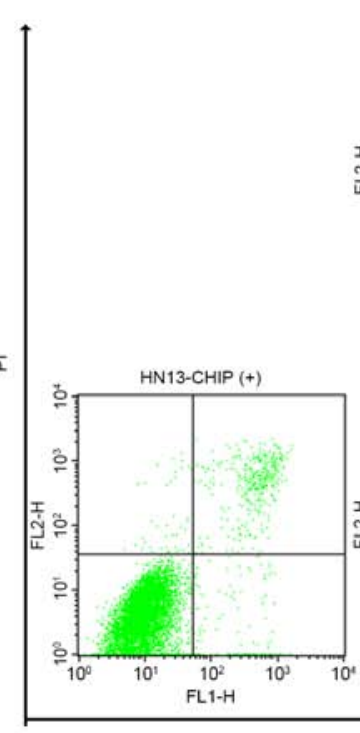

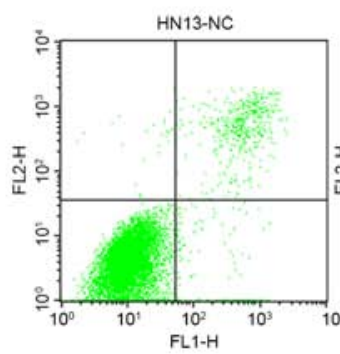
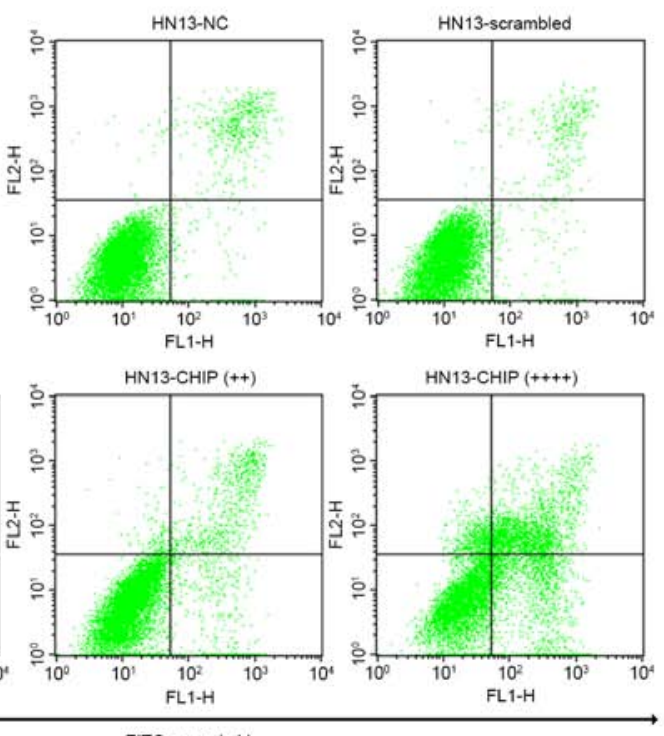

FITC-annexin $V$

Figure 2. Effects of CHIP on migration/invasion capacity and apoptotic status of HNC cells. (A) Transwell assays were conducted to evaluate the effects of CHIP overexpression and knockdown on the migration ability of HN13 and UMSCC12 cells, respectively $\left({ }^{* *} \mathrm{p}<0.01\right)$. (B) The effects of CHIP overexpression and knockdown on the invasion ability of HN13 and UMSCC12 cells were illustrated by Transwell assays, respectively $\left({ }^{* *} \mathrm{p}=0.003\right.$, analysis for the increasing-amount expression of exogenous CHIP in transient transfected HN13 cells. The expression of CHIP was increased to 2- and 4-fold of the regular dose used in the previous assays ( $1.5 \mu \mathrm{g}$ myc-CHIP plasmid in $5 \times 10^{5}$ cells). (D) Flow cytometric analysis for apoptosis reveals that increased expression of CHIP resulted in an increased rate of cellular apoptosis in a dose-dependent manner in HN13 cells. (E) Quantitative analysis and comparison for the percentage of apoptotic cells in CHIP-overexpressing HN13 cells $\left({ }^{* *} \mathrm{p}<0.001\right)$.

lentivirus (Fig. 1D). Likewise, CHIP knockdown promoted the proliferation of UMSCC12 cells (Fig. 1E). The results of colony formation assays revealed that $\mathrm{CHIP}{ }^{\mathrm{OE}}$ significantly decreased the colony formation number (Fig. 1F), which was not observed upon overexpression of the mutant constructs CHIP (K30A) or CHIP (H260Q). The rate of colony formation was significantly enhanced in the UMSCC12shCHIP cells (Fig. 1G).

The biological effects of CHIP on the migration and invasion abilities of HNCs. The migration and invasion abilities of the involved cells were detected by Transwell assays. The 

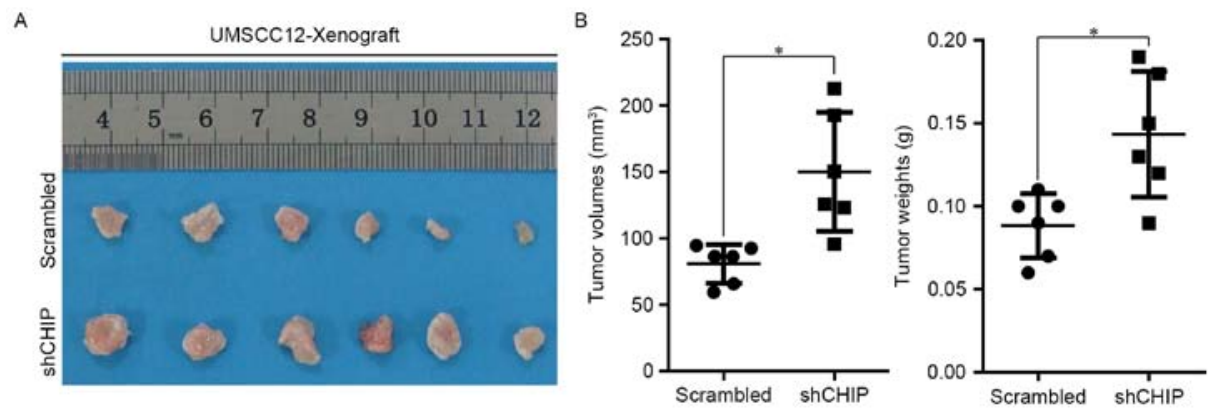

c UMSCC12-Xenograft

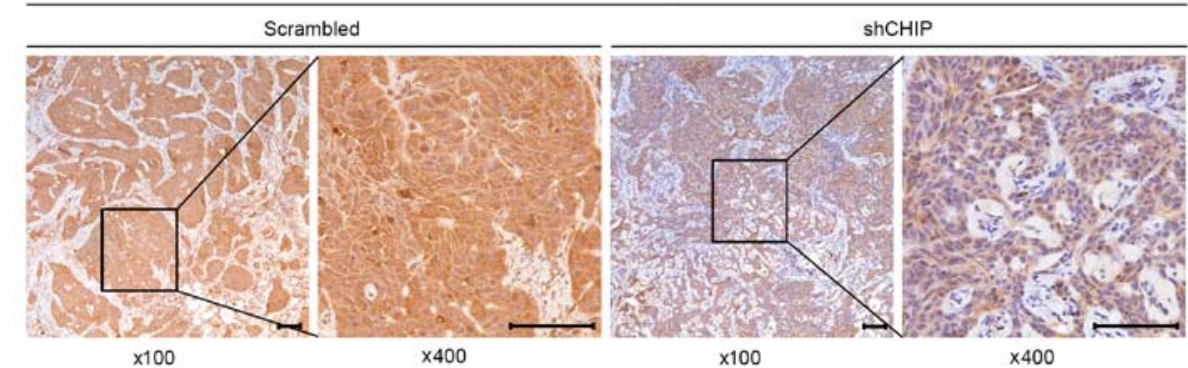

D
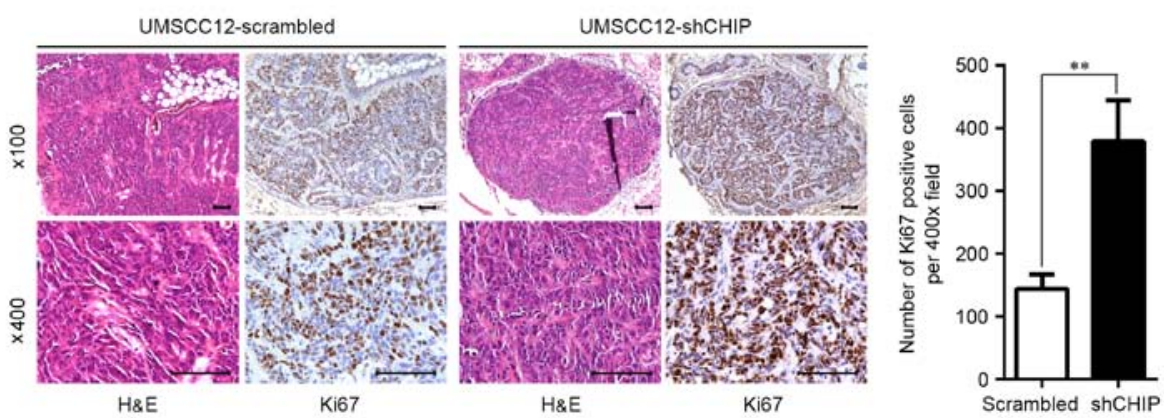

Figure 3. Involvement of CHIP knockdown in the tumorigenesis of HNCs. (A) Representative gross morphology of UMSCC12 xenografts in BALB/c nude mice 60 days after implantation. (B) Tumor volumes and weights for UMSCC12 xenografts ( $\mathrm{*}=0.011$ and ${ }^{*} \mathrm{p}=0.010$, respectively). (C) The expression of CHIP was observed microscopically in UMSCC12-scrambled and UMSCC12-shCHIP xenograft tissues by immunohistochemical staining; scale bar, $100 \mu \mathrm{m}$. (D) H\&E staining and Ki67 immunohistochemistry of consecutive sections of UMSCC12 cell xenografts; scale bar, $100 \mu \mathrm{m}$. The number of Ki67-positive cells $/ \times 400$ fields were counted and compared between the UMSCC12 cell xenografts $(* \mathrm{*} \times 0.01)$.

A

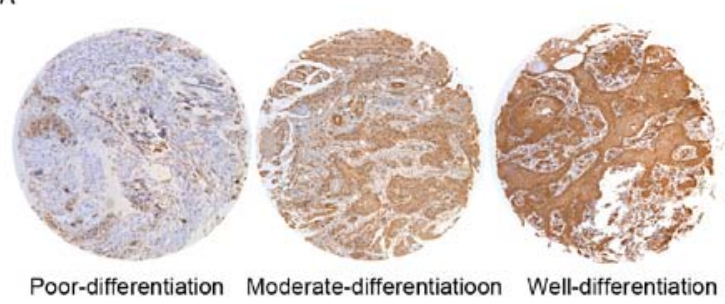

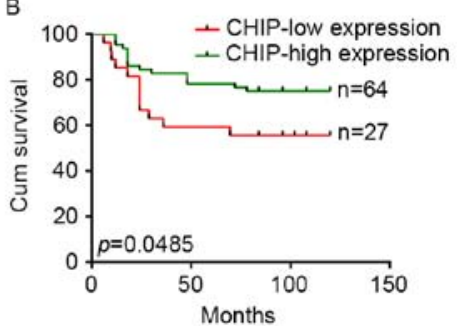

Figure 4. CHIP expression in clinical samples and its clinical significance. (A) Representative immunohistochemical staining of CHIP and the relationship of CHIP expression and differentiation status from an HNC tissue microarray; magnification (×100). (B) Kaplan-Meier analysis of lower CHIP expression shows a significant correlation with poor overall survival (OS) in HNC patients $(\mathrm{p}=0.0485)$.

results indicated that $\mathrm{CHIP} \mathrm{PE}^{\mathrm{OE}}$ caused a dramatic decrease in the migration and invasion abilities of the HN13 cells, whereas the migration and invasion abilities of the UMSCC12shCHIP cells were significantly increased compared with the UMSCC12-scrambled cells (Fig. 2A and B).

Apoptotic induction of CHIP overexpression in HNCs. In HN13 cells, we managed to increase the expression of CHIP to 2- and 4-fold of the regular dose used in the previous assays $\left(1.5 \mu \mathrm{g}\right.$ myc-CHIP plasmid in $5 \times 10^{5}$ cells; Fig. $\left.2 \mathrm{C}\right)$. We observed an increased rate of cellular apoptosis in a dose-dependent manner for CHIP overexpression $48 \mathrm{~h}$ after transfection (Fig. 2D and E; p <0.01), suggesting that CHIP overexpression may serve as an apoptotic inducible factor in HNCs.

Involvement of CHIP knockdown in tumorigenesis of HNCs. To investigate the biological effect of CHIP in the tumorigenesis 
Table I. Demographic characteristics of the patient population according to CHIP expression.

\begin{tabular}{|c|c|c|c|c|}
\hline Variable & $\mathrm{N}$ & $\begin{array}{c}\text { CHIP } \\
\text { expression } \\
\text { (IRS }=1-6) \\
\mathrm{n}(\%)\end{array}$ & $\begin{array}{c}\text { CHIP } \\
\text { expression } \\
\text { (IRS=7-12) } \\
\mathrm{n}(\%)\end{array}$ & P-value \\
\hline All cases & 98 & 28 & 70 & \\
\hline Age, years & & & & 0.602 \\
\hline$<60$ & 59 & $18(64.3)$ & $41(58.6)$ & \\
\hline$\geq 60$ & 39 & $10(35.7)$ & $29(41.4)$ & \\
\hline Sex & & & & 0.337 \\
\hline Male & 52 & 17 (60.7) & $35(50)$ & \\
\hline Female & 46 & $11(39.3)$ & $35(50)$ & \\
\hline Smoking & & & & 0.752 \\
\hline Yes & 32 & $10(41.7)$ & $22(37.9)$ & \\
\hline No & 50 & $14(58.3)$ & $36(62.1)$ & \\
\hline Alcohol & & & & 0.418 \\
\hline Yes & 22 & $8(33.3)$ & 14 (24.6) & \\
\hline No & 59 & $16(66.7)$ & $43(75.4)$ & \\
\hline $\begin{array}{l}\text { Histological grade } \\
\text { (differentiation) }\end{array}$ & & & & 0.000 \\
\hline Well/moderate & 87 & $18(64.3)$ & 69 (98.6) & \\
\hline Poor & 11 & $10(35.7)$ & $1(1.4)$ & \\
\hline Tumor size & & & & 0.808 \\
\hline $\mathrm{T} 1+\mathrm{T} 2$ & 59 & $17(65.4)$ & $42(62.7)$ & \\
\hline $\mathrm{T} 3+\mathrm{T} 4$ & 34 & $9(34.6)$ & $25(37.3)$ & \\
\hline Nodal status & & & & 0.373 \\
\hline No & 67 & $17(65.4)$ & $50(74.6)$ & \\
\hline $\mathrm{N} 1+$ & 26 & $9(34.6)$ & $17(25.4)$ & \\
\hline Metastasis & & & & 1.000 \\
\hline M0 & 92 & $26(100)$ & 66 (98.5) & \\
\hline M1 & 1 & $0(0)$ & $1(1.5)$ & \\
\hline Local recurrence & & & & 1.000 \\
\hline Yes & 5 & $1(3.7)$ & $4(5.8)$ & \\
\hline No & 91 & $26(96.3)$ & $65(94.2)$ & \\
\hline
\end{tabular}

IRS, immunoreactivity score; CHIP, carboxy-terminus Hsc70 interacting protein. $\mathrm{P}$-values in bold print indicate statistical significance.

of HNCs, UMSCC12-scrambled and UMSCC12-shCHIP stable transfected cells were subcutaneously inoculated in the nude mouse. Morphologically, xenograft tumors derived from the UMSCC12-shCHIP cells were larger than the xenograft tumors from the UMSCC12-scrambled cells (Fig. 3A). Quantitatively, UMSCC12-shCHIP cells formed xenografts with significantly larger tumor volume $(\mathrm{p}=0.011)$ and greater tumor mass (Fig. 3B; $\mathrm{p}=0.010$ ). In addition, the subsequent IHC staining further validated the suppressed expression of CHIP in the UMSCC12-shCHIP xenograft specimens (Fig. 3C). Moreover, we observed significantly increased Ki67-positive cells, a marker for proliferation, in the UMSCC12-shCHIP xenografts compared with this number in the UMSCC12scrambled xenografts (Fig. 3D). These results suggest that expression of CHIP negatively affects the malignant characteristics of HNC cell lines.

Suppression of CHIP expression acts as a risk factor for $H N C$ patients. In order to illustrate the clinical significance of variable CHIP expression in HNCs, a retrospective cohort was included and analyzed with matched tissue array. The IHC analysis of clinical samples revealed that high CHIP expression occurred in 70 of $98 \mathrm{HNC}$ samples. In the included cohort, the CHIP expression level was significantly associated with the differentiation status of cancer cells, whereas no significance was observed for the other parameters (Table I). As shown in Fig. 4A, we observed a changing expression pattern of CHIP from poor, moderate, to well differentiation pathological status in the HNC specimens. Since 7 samples lacked follow-up data, the effect of CHIP expression on the overall survival (OS) was analyzed in 91 cases. Patients with HNC cancers that expressed lower CHIP showed poorer OS ( $\mathrm{p}=0.0485$; Fig. 4B).

\section{Discussion}

In the present study, we illustrated the inhibition of cancer cell growth by the E3 ligase CHIP in HNCs with a series of in vitro and in vivo assays. In HNC clinical samples, CHIP expression was shown to be significantly related to the differentiation status of the HNCs, and low expression of CHIP indicated poorer OS in HNC patients.

Increasing evidence strongly suggests that ubiquitin-dependent proteolysis, mediated by the E3 ligase CHIP, plays an essential role in regulating various biological processes during carcinogenesis. Previously, we reviewed the reported studies on the biological effects of CHIP on cancers by searching PubMed database up to December, 2016. In summary, both oncogene and tumor suppressor functions of CHIP have been reported in variant cancers by regulating underlying targets $(2,7,8,19)$. In addition, the E3 ligase CHIP has been illustrated to exert its biological functions in cancer by targeting more than 30 types of proteins (data not shown). In breast cancer, the oncogene effects of CHIP were reported by regulating PTEN and Pfn1 $(15,20,21)$. However, the tumor suppressor effects of CHIP were reported by targeting TRAF2, ErbB2, ER $\alpha$, MIF, PTK6, SRC-3, CtBP2 (7,22-29). However, in prostate cancer, CHIP was reported as an oncogene by targeting PTEN and Mst1, and as a tumor suppressor by targeting AR $(16,19,30)$. The production of mutated proteins or abnormally expressed proteins seems to be unavoidable during carcinogenesis. Undoubtedly, the biological functions of CHIP in each cancer type may not only target just one specific protein. Thus, it seems to be more meaningful to investigate the biological effect of E3 ligase CHIP on certain behaviors of cancer cells. In our previous studies, we illustrated that CHIP regulated the cancer stem-like properties of HNCs by targeting CD166 protein (31).

Previous studies have reported that CHIP functions as a tumor-suppressor in pancreatic, colorectal and gastric cancer (12-14,23). In pancreatic cancer, CHIP was reported to impair cell proliferation, migration and invasion by targeting EGFR (12). In colorectal cancer, CHIP impaired tumor growth, migration and invasion by repressing NF- $\mathrm{kB}$-mediated 
signaling (13). In breast cancer cells, anti-apoptotic protein Bcl-2 was downregulated by CHIP (32). Furthermore, CHIP was reported to modulate mitotic arrest by degradation of the androgen receptor and c-Myc $(30,33)$. In the present study, we identified the function of CHIP as a candidate tumor suppressor using a series of in vitro and in vivo assays. We found that altered CHIP expression regulated the cellular proliferation, migration/invasion abilities and tumor growth in HNCs. However, overexpression of CHIP induced increased apoptotic levels in HNC cells. In HNC samples, low expression of CHIP indicated a poor differentiation status and a higher risk of reduced OS. Accordingly, we conclude that suppressed expression of CHIP participates in the progression of HNCs. Herein, the underlying targets of CHIP were not further investigated and further studies are warranted to investigate the underlying mechanisms.

CHIP is a cochaperone E3 ligase containing 3 TPR motifs and a U-box domain (34). In the present study, we managed to construct two loss-of-function mutants of CHIP at the TPR motifs and U-box domain, respectively. Expectedly, these two mutants abolished the suppressive function of wildtype CHIP protein in regards to the proliferative and colony forming abilities of $\mathrm{HNCs}$, indicating that the biological effects of CHIP in HNCs were TPR motif- and U-box domain-dependent. Functionally, CHIP has been identified as being associated with Hsc70 and Hsp90 to achieve the subsequent ubiquitination of targeting protein via the U-box domain (35). Accordingly, the inhibition of Hsp90 by 17-AAG or 17-DMAG was found to increase the expression levels of Hsp70 and Hsp90, resulting in enhanced CHIP-mediated ubiquitination and the following proteasomal degradation $(10,36,37)$. Hsp90-targeted therapy has been advanced and well documented and has been proposed as a prospective treatment method in various types of cancers $(38,39)$. Above all, to enhance the tumor suppressor functions of CHIP in HNCs using an Hsp90 inhibitor may be a new treatment strategy for HNC patients.

In conclusion, the present study demonstrated that CHIP functions as a candidate tumor suppressor in HNCs. Meanwhile, we demonstrated that suppression of the expression of CHIP may participate in the development and progression of HNCs. Thus, identifying a strategy by which to increase the expression or to enhance the biological function of CHIP may benefit HNC patients in clinical practice.

\section{Acknowledgements}

We thank Professor Zeguang Han, Professor Li Mao and Wenyi Wei for providing insightful comments for the present study. We also thank members of the Chenping Zhang's Department of Oral and Maxillofacial Head and Neck Oncology for providing the clinical information of the involved HNC specimens in the present study. The present study was supported by the National Program on Key Research Project of China (2016YFC0902700), the National Natural Science Foundation of China (81272978 and 91229103), the Project of the Shanghai Science and Technology Committee (15DZ22992200) and the Innovation Fund for Doctoral Program of Shanghai Jiaotong University, School of Medicine (BXJ201628).

\section{References}

1. Kumar P, Pradhan K, Karunya R, Ambasta RK and Querfurth HW: Cross-functional E3 ligases Parkin and C-terminus $\mathrm{Hsp} 70$-interacting protein in neurodegenerative disorders. J Neurochem 120: 350-370, 2012.

2. Yan S, Sun X, Xiang B, Cang H, Kang X, Chen Y, Li H, Shi G, Yeh ET, Wang B, et al: Redox regulation of the stability of the SUMO protease SENP3 via interactions with CHIP and Hsp90. EMBO J 29: 3773-3786, 2010.

3. Seo J, Lee EW, Sung H, Seong D, Dondelinger Y, Shin J, Jeong M, Lee HK, Kim JH, Han SY, et al: CHIP controls necroptosis through ubiquitylation- and lysosome-dependent degradation of RIPK3. Nat Cell Biol 18: 291-302, 2016.

4. Paul I and Ghosh MK: The E3 ligase CHIP: Insights into its structure and regulation. Biomed Res Int 2014: 918183, 2014.

5. Edkins AL: CHIP: A co-chaperone for degradation by the proteasome. Subcell Biochem 78: 219-242, 2015.

6. Wei Q, Sha Y, Bhattacharya A, Abdel Fattah E, Bonilla D, Jyothula SS, Pandit L, Khurana Hershey GK and Eissa NT: Regulation of IL-4 receptor signaling by STUB1 in lung inflammation. Am J Respir Crit Care Med 189: 16-29, 2014.

7. Kajiro M, Hirota R, Nakajima Y, Kawanowa K, So-ma K, Ito I, Yamaguchi Y, Ohie SH, Kobayashi Y, Seino Y, et al: The ubiquitin ligase CHIP acts as an upstream regulator of oncogenic pathways. Nat Cell Biol 11: 312-319, 2009.

8. Gaude H, Aznar N, Delay A, Bres A, Buchet-Poyau K, Caillat C, Vigouroux A, Rogon C, Woods A, Vanacker JM, et al: Molecular chaperone complexes with antagonizing activities regulate stability and activity of the tumor suppressor LKB1. Oncogene 31: 1582-1591, 2012.

9. Hatakeyama S, Watanabe M, Fujii Y and Nakayama KI: Targeted destruction of c-Myc by an engineered ubiquitin ligase suppresses cell transformation and tumor formation. Cancer Res 65: 7874-7879, 2005.

10. Ruckova E, Muller P, Nenutil R and Vojtesek B: Alterations of the Hsp70/Hsp90 chaperone and the HOP/CHIP co-chaperone system in cancer. Cell Mol Biol Lett 17: 446-458, 2012.

11. Ciocca DR and Calderwood SK: Heat shock proteins in cancer: Diagnostic, prognostic, predictive, and treatment implications. Cell Stress Chaperones 10: 86-103, 2005.

12. Wang T, Yang J, Xu J, Li J, Cao Z, Zhou L, You L, Shu H, Lu Z, $\mathrm{Li} \mathrm{H}$, et al: CHIP is a novel tumor suppressor in pancreatic cancer through targeting EGFR. Oncotarget 5: 1969-1986, 2014.

13. Wang Y, Ren F, Wang Y, Feng Y, Wang D, Jia B, Qiu Y, Wang S, Yu J, Sung JJ, et al: CHIP/Stub1 functions as a tumor suppressor and represses NF- $\mathrm{BB}$-mediated signaling in colorectal cancer. Carcinogenesis 35: 983-991, 2014.

14. Wang S, Wu X, Zhang J, Chen Y, Xu J, Xia X, He S, Qiang F, Li A, Shu Y, et al: CHIP functions as a novel suppressor of tumour angiogenesis with prognostic significance in human gastric cancer. Gut 62: 496-508, 2013.

15. Ying Z, Haiyan G and Haidong G: BAG5 regulates PTEN stability in MCF-7 cell line. BMB Rep 46: 490-494, 2013.

16. Ahmed SF, Deb S, Paul I, Chatterjee A, Mandal T, Chatterjee U and Ghosh MK: The chaperone-assisted $\mathrm{E} 3$ ligase $\mathrm{C}$ terminus of Hsc70-interacting protein (CHIP) targets PTEN for proteasomal degradation. J Biol Chem 287: 15996-16006, 2012.

17. Yan M, Yang X, Wang L, Clark D, Zuo H, Ye D, Chen W and Zhang P: Plasma membrane proteomics of tumor spheres identify CD166 as a novel marker for cancer stem-like cells in head and neck squamous cell carcinoma. Mol Cell Proteomics 12: 3271-3284, 2013.

18. Lv Z, Wu X, Cao W, Shen Z, Wang L, Xie F, Zhang J, Ji T, Yan M and Chen W: Parathyroid hormone-related protein serves as a prognostic indicator in oral squamous cell carcinoma. J Exp Clin Cancer Res 33: 100, 2014.

19. Ren A, Yan G, You B and Sun J: Down-regulation of mammalian sterile 20 -like kinase 1 by heat shock protein 70 mediates cisplatin resistance in prostate cancer cells. Cancer Res 68: 2266-2274, 2008.

20. Lv Y, Song S, Zhang K, Gao H and Ma R: CHIP regulates AKT/FoxO/Bim signaling in MCF7 and MCF10A cells. PLoS One 8: e83312, 2013.

21. Choi YN, Lee SK, Seo TW, Lee JS and Yoo SJ: C-Terminus of Hsc70-interacting protein regulates profilin1 and breast cancer cell migration. Biochem Biophys Res Commun 446: 1060-1066, 2014. 
22. Jang KW, Lee KH, Kim SH, Jin T, Choi EY, Jeon HJ, Kim E, Han YS and Chung JH: Ubiquitin ligase CHIP induces TRAF2 proteasomal degradation and $\mathrm{NF}-\kappa \mathrm{B}$ inactivation to regulate breast cancer cell invasion. J Cell Biochem 112: 3612-3620, 2011.

23. Jan CI, Yu CC, Hung MC, Harn HJ, Nieh S, Lee HS, Lou MA Wu YC, Chen CY, Huang CY, et al: Tid1, CHIP and ErbB2 interactions and their prognostic implications for breast cancer patients. J Pathol 225: 424-437, 2011.

24. Yi X, Wei W, Wang SY, Du ZY, Xu YJ and Yu XD: Histone deacetylase inhibitor SAHA induces ERalpha degradation in breast cancer MCF-7 cells by CHIP-mediated ubiquitin pathway and inhibits survival signaling. Biochem Pharmacol 75: 1697-1705, 2008

25. Raja SM, Clubb RJ, Bhattacharyya M, Dimri M, Cheng H, Pan W, Ortega-Cava C, Lakku-Reddi A, Naramura M, Band V, et al: A combination of Trastuzumab and 17-AAG induces enhanced ubiquitinylation and lysosomal pathway-dependent ErbB2 degradation and cytotoxicity in ErbB2-overexpressing breast cancer cells. Cancer Biol Ther 7: 1630-1640, 2008.

26. Kang SA, Cho HS, Yoon JB, Chung IK and Lee ST: Hsp90 rescues PTK6 from proteasomal degradation in breast cancer cells. Biochem J 447: 313-320, 2012.

27. Jeong JH, An JY, Kwon YT, Li LY and Lee YJ: Quercetininduced ubiquitination and down-regulation of Her-2/neu. J Cell Biochem 105: 585-595, 2008

28. Lee JS and Yoo SJ: C-terminus of Hsc70-interacting protein regulates $\mathrm{C}$-terminal binding protein 2 and the expression of its target genes. Biochem Biophys Res Commun 432: 418-424, 2013

29. Schulz R, Marchenko ND, Holembowski L, Fingerle-Rowson G, Pesic M, Zender L, Dobbelstein M and Moll UM: Inhibiting the HSP90 chaperone destabilizes macrophage migration inhibitory factor and thereby inhibits breast tumor progression. J Exp Med 209: 275-289, 2012.

30. Sarkar S, Brautigan DL, Parsons SJ and Larner JM: Androgen receptor degradation by the E3 ligase CHIP modulates mitotic arrest in prostate cancer cells. Oncogene 33: 26-33, 2014.

31. Xiao M, Yan M, Zhang J, Xu Q, Qi S, Wang X and Chen W: Cancer stem-like cell related protein CD166 degrades through E3 ubiquitin ligase CHIP in head and neck cancer. Exp Cell Res 353: 46-53, 2017.
32. Tsuchiya M, Nakajima Y, Waku T, Hiyoshi H, Morishita T, Furumai R, Hayashi Y, Kishimoto H, Kimura $K$ and Yanagisawa J: CHIP buffers heterogeneous Bcl-2 expression levels to prevent augmentation of anticancer drug-resistant cell population. Oncogene 34: 4656-4663, 2015.

33. Paul I, Ahmed SF, Bhowmik A, Deb S and Ghosh MK: The ubiquitin ligase CHIP regulates c-Myc stability and transcriptional activity. Oncogene 32: 1284-1295, 2013.

34. McDonough $\mathrm{H}$ and Patterson C: CHIP: A link between the chaperone and proteasome systems. Cell Stress Chaperones 8: 303-308, 2003.

35. Murata S, Chiba T and Tanaka K: CHIP: A quality-control E3 ligase collaborating with molecular chaperones. Int J Biochem Cell Biol 35: 572-578, 2003.

36. Tsuchiya M, Nakajima Y, Hirata N, Morishita T, Kishimoto H, Kanda Y and Kimura K: Ubiquitin ligase CHIP suppresses cancer stem cell properties in a population of breast cancer cells. Biochem Biophys Res Commun 452: 928-932, 2014.

37. Muller P, Ruckova E, Halada P, Coates PJ, Hrstka R, Lane DP and Vojtesek B: C-terminal phosphorylation of Hsp70 and Hsp90 regulates alternate binding to co-chaperones CHIP and HOP to determine cellular protein folding/degradation balances. Oncogene 32: 3101-3110, 2013

38. Ghadban T, Jessen A, Reeh M, Dibbern JL, Mahner S, Mueller V, Wellner UF, Güngör C, Izbicki JR and Vashist YK: In vitro study comparing the efficacy of the water-soluble HSP90 inhibitors, 17-AEPGA and 17-DMAG, with that of the nonwater-soluble HSP90 inhibitor, 17-AAG, in breast cancer cell lines. Int J Mol Med 38: 1296-1302, 2016.

39. Haque A, Alam Q, Alam MZ, Azhar EI, Sait KH, Anfinan N, Mushtaq G, Kamal MA and Rasool M: Current understanding of HSP90 as a novel therapeutic target: An emerging approach for the treatment of cancer. Curr Pharm Des 22: 2947-2959, 2016. 